\title{
Article \\ Extra Virgin Oil Polyphenols Improve the Protective Effects of Hydroxytyrosol in an In Vitro Model of Hypoxia-Reoxygenation of Rat Brain
}

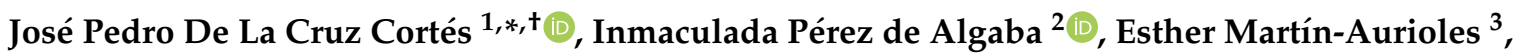 \\ María Monsalud Arrebola ${ }^{4}$ (D) Laura Ortega-Hombrados ${ }^{1}$, María Dolores Rodríguez-Pérez ${ }^{1}$, \\ María África Fernández-Prior ${ }^{5}$ (D), Alejandra Bermúdez-Oria ${ }^{5}{ }^{(0)}$, Cristina Verdugo ${ }^{1}$ \\ and José Antonio González-Correa ${ }^{1}$
}

check for

updates

Citation: De La Cruz Cortés, J.P.;

Pérez de Algaba, I.; Martín-Aurioles,

E.; Arrebola, M.M.;

Ortega-Hombrados, L.;

Rodríguez-Pérez, M.D.;

Fernández-Prior, M.Á.;

Bermúdez-Oria, A.; Verdugo, C.;

González-Correa, J.A. Extra Virgin

Oil Polyphenols Improve the

Protective Effects of Hydroxytyrosol

in an In Vitro Model of

Hypoxia-Reoxygenation of Rat Brain.

Brain Sci. 2021, 11, 1133. https://

doi.org/10.3390/brainsci11091133

Academic Editor: Ashu Johri

Received: 22 July 2021

Accepted: 24 August 2021

Published: 26 August 2021

Publisher's Note: MDPI stays neutral with regard to jurisdictional claims in published maps and institutional affiliations.

Copyright: (c) 2021 by the authors. Licensee MDPI, Basel, Switzerland. This article is an open access article distributed under the terms and conditions of the Creative Commons Attribution (CC BY) license (https:// creativecommons.org/licenses/by/ $4.0 /)$.
1 Departmento de Farmacología, Facultad de Medicina, Instituto de Investigación Biomédica (IBIMA), Universidad de Málaga, 29010 Málaga, Spain; lauraortegah@outlook.es (L.O.-H.); mariaperezrodriguez@ymail.com (M.D.R.-P.); cristinaverdugocabello@gmail.com (C.V.); correa@uma.es (J.A.G.-C.)

2 Clinical Laboratory Department, Hospital Público de Montilla, 14550 Córdoba, Spain; iperezdealgaba@gmail.com

3 UGC La Roca, Distrito Sanitario Málaga-Guadalhorce, 29001 Málaga, Spain; esther.uma@hotmail.com

4 UGC Laboratorio Clínico, Hospital de la Axarquía, AGSEMA, 29740 Málaga, Spain; mariam.arrebola.sspa@juntadeandalucia.es

5 Instituto de la Grasa, Consejo Superior de Investigaciones Científicas (CSIC), Ctra. Utrera Km 1, Campus Universitario Pablo de Olavide, Edificio 46, 41013 Seville, Spain; mafprior@ig.csic.es (M.Á.F.-P.); aleberori@ig.csic.es (A.B.-O.)

* Correspondence: jpcruz@uma.es; Tel.: +34-952-131-567

+ Current address: Department of Pharmacology, School of Medicine, Campus de Teatinos s/n, University of Málaga, 29010 Málaga, Spain.

Abstract: Hydroxytyrosol (HT) is the component primarily responsible for the neuroprotective effect of extra virgin olive oil (EVOO). However, it is less effective on its own than the demonstrated neuroprotective effect of EVOO, and for this reason, it can be postulated that there is an interaction between several of the polyphenols of EVOO. The objective of the study was to assess the possible interaction of four EVOO polyphenols (HT, tyrosol, dihydroxyphenylglycol, and oleocanthal) in an experimental model of hypoxia-reoxygenation in rat brain slices. The lactate dehydrogenase (LDH) efflux, lipid peroxidation, and peroxynitrite production were determined as measures of cell death, oxidative stress, and nitrosative stress, respectively. First, the polyphenols were incubated with the brain slices in the same proportions that exist in EVOO, comparing their effects with those of HT. In all cases, the cytoprotective and antioxidant effects of the combination were greater than those of HT alone. Second, we calculated the concentration-effect curves for HT in the absence or presence of each polyphenol. Tyrosol did not significantly modify any of the variables inhibited by HT. Dihydroxyphenylglycol only increased the cytoprotective effect of HT at $10 \mu \mathrm{M}$, while it increased its antioxidant effect at 50 and $100 \mu \mathrm{M}$ and its inhibitory effect on peroxynitrite formation at all the concentrations tested. Oleocanthal increased the cytoprotective and antioxidant effects of HT but did not modify its inhibitory effect on nitrosative stress. The results of this study show that the EVOO polyphenols DHPG and OLC increase the cytoprotective effect of HT in an experimental model of hypoxia-reoxygenation in rat brain slices, mainly due to a possibly synergistic effect on HT's antioxidant action. These results could explain the greater neuroprotective effect of EVOO than of the polyphenols alone.

Keywords: hydroxytyrosol; extra virgin olive oil; polyphenols; neuroprotection 


\section{Introduction}

The beneficial effect of the intake of extra virgin olive oil (EVOO) for the prevention of cardiovascular diseases has been widely demonstrated [1]. High adherence to a Mediterranean diet model supplemented with approximately $50 \mathrm{~mL}$ of EVOO per day has been shown to reduce the incidence of coronary ischemic attacks, peripheral artery disease, and ischemic stroke, in addition to preventing the onset of obesity and type 2 diabetes [1,2].

In an experimental study, the neuroprotective effect of the administration of doses of EVOO equivalent to those recommended for humans was demonstrated, both in normal rats and in an experimental model of type 1 diabetes [3]. Fundamentally, it has been shown that, in an in vitro model of hypoxia-reoxygenation in the brain slices of rats, chronic treatment with EVOO results in a significantly lower rate of cell death $[3,4]$.

Likewise, different chemical components of EVOO have been studied separately, and the cardioprotective and neuroprotective effects of EVOO have been attributed to the polyphenol most abundant in its composition, hydroxytyrosol (HT), [5]. These effects are explained by the antioxidant effect demonstrated for HT in various tissues and in experimental models of disease, such as diabetes, hypertension, and dyslipidemia [6,7].

When the different polyphenols were studied separately, none of them could fully explain the effect exerted by EVOO [8]. Specifically, in terms of its neuroprotective effect, HT is the compound that can best explain the effect of EVOO, but it does not do so entirely [8]. For this reason, taking into account the other polyphenols in the composition of EVOO $[9,10]$, it could be postulated that there is an interaction between them when they reach nervous tissue and thus, together they exert the total observed effects of EVOO.

In this study, we investigated four of the main polyphenols present in EVOO: hydroxytyrosol (HT), tyrosol (Ty), 3', 4'-dihydroxyphenylglycol (DHPG), and oleocanthal (OLC). We chose these four EVOO compounds because they are recognized as simple polyphenols with more biological potency than others in EVOO [6]. Some others, such as oleuropein, act by way of their HT content; HT is released in the stomach when oleuropein is ingested orally. Hydroxytyrosol is considered to have the highest biological activity of all the polyphenols in EVOO [8]. The objective of this study was to assess the possible effects that Ty, DHPG, and OLC could exert on the in vitro cytoprotective, antioxidant, and antinitrosative effects of HT in an experimental model of hypoxia-reoxygenation in rat brain slices.

\section{Materials and Methods}

\subsection{Materials}

A thiobarbituric acid reactive substances colorimetric kit and 3-nitrotyrosine enzyme immunoassay kits were obtained from Cell Biolabs Inc. (Bionova Científica S.L., Madrid, Spain). A lactate dehydrogenase colorimetric kit (Cytotoxicity Detection Kit) was obtained from Roche Applied Science (Barcelona, Spain). All the other reagents, including the tyrosol and oleocanthal, were from Sigma Chemical Corp. (St. Louis, MO, USA).

Hydroxytyrosol was isolated by a hydrothermal treatment of the liquid phase obtained from alperujo (a by-product of the two-phase olive oil separation system) at $160{ }^{\circ} \mathrm{C}$ for $60 \mathrm{~min}$ [11]. The liquid was extracted by two-step chromatography fractionation. The final yield reached $99.6 \%$ purity relative to dry matter, using the process described by Fernández-Bolaños et al. [12]. The phenols were quantified using a Hewlett-Packard 1100 liquid chromatography system with an ultraviolet-visible detector. A Mediterranean Sea C18 analytical column $(250 \times 4.6 \mathrm{~mm}$ i.d.; particle size $=5 \mu \mathrm{m})($ Teknokroma, Barcelona, Spain) was used at room temperature. The system was equipped with Rheodyne injection valves $(20 \mu \mathrm{L}$ loop). The mobile phases were $0.01 \%$ trichloroacetic acid in water and acetonitrile, with the following gradient during a total run time of $55 \mathrm{~min}$ : 95\% initially, $75 \%$ at $30 \mathrm{~min}, 50 \%$ at $45 \mathrm{~min}, 0 \%$ at $47 \mathrm{~min}, 75 \%$ at $50 \mathrm{~min}$, and $95 \%$ at $52 \mathrm{~min}$ until the run was complete. Quantification was carried out by peak integration at a $280 \mathrm{~nm}$ wavelength with reference to the calibrations obtained with external standards (Figure 1). 


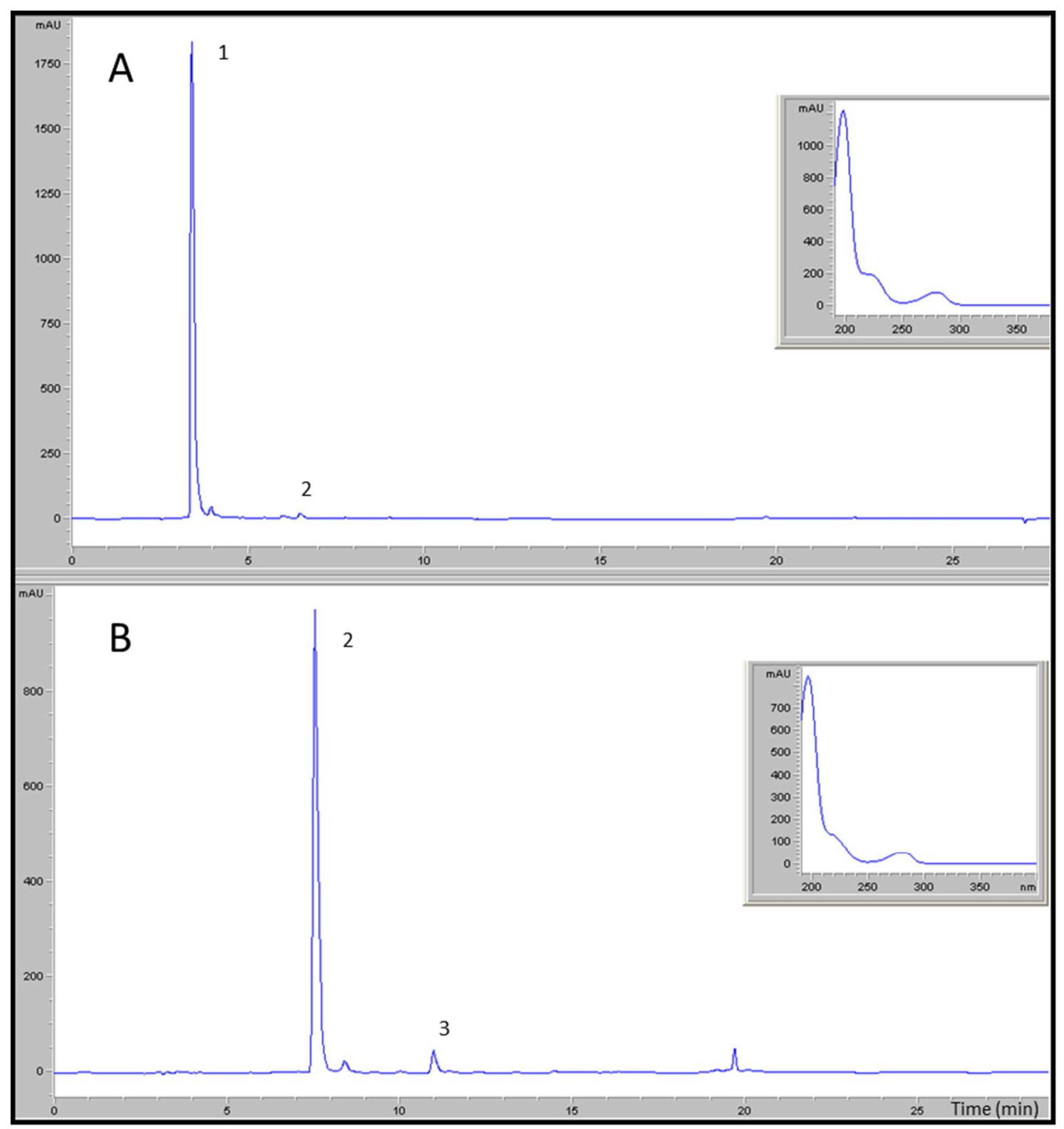

Figure 1. Chromatographic profiles at $280 \mathrm{~nm}$ of the purified 3,4-dihydroxyphenylglycol (A) and purified hydroxytyrosol (B) obtained from the HPLC-DAD and their UV-visible absorption spectra. The main compounds detected were (1) 3,4-dihydroxyphenylglycol, (2) hydroxytyrosol at 99.6\%, and (3) tyrosol.

$3^{\prime}, 4^{\prime}$-dihidroxifenilglicol, obtained from the olive oil by-product of the two-phase extraction system used in olive oil mills, was used for the isolation of the DHPG. The method for purifying DHPG was described and patented by Fernández-Bolaños et al. (2010) (WO2010070168A1). The method is based on physical chromatographic systems that allow for the extraction of natural compounds without any organic solvent or chemical or enzymatic reactions, realizing a purity over $95 \%$, with reference to dry matter (Figure 1).

\subsection{Study Design}

The animals were two-month-old adult male Wistar rats (with body weights of 200-250 g). All the rats were used in accordance with the current Spanish legislation for animal care, use, and housing (EDL 2013/80847, BOE-A-2013-6271). The recommendations of the Guide for the Care and Use of Laboratory Animals (NIH publication No. 86-23, revised 1985) were followed, as was the Spanish Law on the Protection of Animals, where applicable. The study protocol was approved by the University of Malaga Ethics Commit- 
tee for the Use of Animals (Ref. CEUMA31-2018-A) and the Consejería de Agricultura, Ganadería, Pesca y Desarrollo Sostenible, Junta de Andalucía (Department of Agriculture, Livestock, Fisheries and Sustainable Development of the Regional Government of Andalusia) (Ref. 9/07/2019/124).

\subsection{Hypoxia-Reoxygenation Procedure}

All the rats were anesthetized with pentobarbital sodium $(40 \mathrm{mg} / \mathrm{kg}$ i.p.) and then decapitated with a guillotine. The brain tissue (except the cerebellum and brain stem) was cut transversally into $1 \mathrm{~mm}$ slices with a vibrating microtome (Campden Instruments, San Francisco, CA, USA). The slices were placed in a buffer (composition in mmol/L: $100 \mathrm{NaCl}$, $0.05 \mathrm{KCl}, 24 \mathrm{NaHCO}_{3}, 0.55 \mathrm{KH}_{2} \mathrm{PO}_{4}, 0.005 \mathrm{CaCl}_{2}, 2 \mathrm{MgSO}_{4}, 9.8$ glucose, $\mathrm{pH} 7.4$ ) and perfused with a mixture of $95 \% \mathrm{O}_{2}$ and $5 \% \mathrm{CO}_{2}$ (Period A). After $30 \mathrm{~min}$, the brain slices were placed in fresh buffer with no glucose and $3 \mathrm{mmol} / \mathrm{L}$ of $\mathrm{CaCl}_{2}$ and $0.001 \mathrm{mmol} / \mathrm{L}$ of $\mathrm{MgSO}_{4}$. A mixture of $95 \% \mathrm{~N}_{2}$ and $5 \% \mathrm{CO}_{2}$ (hypoxia) was perfused for $20 \mathrm{~min}$ (Period B). Subsequently, the slices were placed in a buffer with glucose and perfused with a mixture of $95 \% \mathrm{O}_{2}$ and $5 \% \mathrm{CO}_{2}$ for $120 \mathrm{~min}$ (reoxygenation) (Period C).

The brain samples were analyzed before the period of hypoxia, after $20 \mathrm{~min}$ of hypoxic incubation, and after $120 \mathrm{~min}$ of reoxygenation. They were then frozen in liquid nitrogen and stored at $-80^{\circ} \mathrm{C}$. All the analytical techniques were performed not more than 7 days after the samples were frozen.

\subsection{Incubation of Polyphenol Compounds}

Four of the main EVOO polyphenol compounds were tested. Hydroxytyrosol (HT) was considered the main compound, and tyrosol (Ty), 3', $4^{\prime}$-dihidroxifenilglicol (DHPG), and oleocanthal (OLC) were studied as possible modifiers of HT's effects.

All the compounds were added from the beginning of the experiment (Period A) and kept until the end of the reoxygenation period (Period C). The concentrations of each compound were chosen according to the ranges of concentrations described for EVOO, with low, medium, and high polyphenol contents $[13,14]$, as well as the effects demonstrated in some in vitro experiments in rat brain $[8,15]$. Table 1 shows the ranges of each compound according to the literature and the exact concentrations incubated in the experiments.

Table 1. Concentration ranges (mg/kg) of hydroxytyrosol (HT), tyrosol (Ty), $3^{\prime}, 4^{\prime}$-dihydroxyphenylglycol (DHPG), and oleocanthal (OLC) in extra virgin olive oils, considered to have low (LP), medium (MP), and high (HP) polyphenol contents.

\begin{tabular}{ccccc}
\hline- & HT & Ty & DHPG & OLC \\
\hline EVOO-LP & - & - & - & - \\
Range & $1.0-5.0$ & - & $0.0-0.5$ & $10-15$ \\
(incubated) & 2.5 & 0.25 & 0.25 & 20 \\
EVOO-MP & - & - & - & - \\
Range & $10-50$ & $0.5-1.1$ & $1.0-5.0$ & $100-200$ \\
(incubated) & 30 & 0.8 & 0.3 & 150 \\
EVOO-HP & - & - & - & - \\
Range & $100-300$ & $2.0-8.0$ & $10-25$ & $250-400$ \\
(incubated) & 200 & 5.0 & 20 & 325 \\
\hline
\end{tabular}

These data have been weighted from the references $[13,14]$. The concentration of each polyphenol that was chosen in the in vitro experiments is indicated as (incubated).

\subsection{Analytical Techniques}

All the techniques were carried out in a single-blind manner; that is, the persons who conducted the assays were unaware of the origin and nature of the samples.

\subsubsection{Lactate Dehydrogenase (LDH) Efflux}

The LDH efflux in the incubation buffer was measured as an indirect representation of cell death. Enzyme activity was measured spectrophotometrically at $340 \mathrm{~nm}$ according 
to the manufacturer's instructions. Briefly, in a 96-well microplate, $100 \mu \mathrm{L}$ of the sample was incubated with $100 \mu \mathrm{L}$ of a reaction solution containing iodotetrazolium chloride and sodium lactate. The incubation of the free buffer with the reaction solution was used as a blank. The plate was incubated for $30 \mathrm{~min}$, and then, $50 \mu \mathrm{L}$ of the stop solution was added. The optical absorbance at $490 \mathrm{~nm}$ and $600 \mathrm{~nm}$ was determined. The value obtained at $600 \mathrm{~nm}$ was subtracted from that obtained at $490 \mathrm{~nm}$, and subsequently, the absorbance obtained for the blank was subtracted from all the values.

\subsubsection{Lipid Peroxidation}

The brain tissue was homogenized in $50 \mathrm{mM}$ phosphate-buffered saline with a $\mathrm{pH}$ of $7.0(1 / 15 \mathrm{w} / \mathrm{v})$. The resulting sample was centrifuged at $13,000 \times \mathrm{g}$ for $15 \mathrm{~min}$ at $4{ }^{\circ} \mathrm{C}$, the supernatant was separated, and aliquots were frozen at $-80{ }^{\circ} \mathrm{C}$ until used for the measurement of lipid peroxidation.

Thiobarbituric acid reactive substances (TBARS) were measured as an index of lipid peroxides, whose main compound is malondialdehyde (MDA). Briefly, $100 \mu \mathrm{L}$ of the samples or increasing concentrations of malondialdehyde were incubated at room temperature for $5 \mathrm{~min}$. Subsequently, $250 \mu \mathrm{L}$ of thiobarbituric acid $(5.2 \mathrm{mg} / \mathrm{mL}, \mathrm{pH} 3.5$, buffered with sodium hydroxide) was added. All the samples were incubated at $95{ }^{\circ} \mathrm{C}$ for $45 \mathrm{~min}$ and centrifuged at $3000 \times g$ for $15 \mathrm{~min}$, and the optical absorbance at $532 \mathrm{~nm}$ of the supernatant was determined in a 96-well plate. The equation of the malondialdehyde standard curve was calculated, and the data obtained for the brain samples were interpolated.

\subsubsection{3-Nitrotyrosine}

As an index of peroxynitrite formation, the concentration of 3-nitrotyrosine was measured. The brain slices were homogenized (1:10 wt/vol) in $100 \mathrm{mM} \mathrm{KH} \mathrm{PO}_{4} / \mathrm{K}_{2} \mathrm{HPO}_{4}$ and $0.1 \%$ digitonin ( $\mathrm{pH}$ of 7.4$)$. Then, they were centrifuged $\left(5000 \times g, 10 \mathrm{~min}, 4{ }^{\circ} \mathrm{C}\right)$. The concentration of 3-nitrotyrosine in the supernatant was measured according to the manufacturer's instructions for the enzyme immunoassay kit. Briefly, in a 96-well plate, $50 \mu \mathrm{L}$ of sample and increasing concentrations of the 3-NTy standard or buffer (blank) were incubated with $50 \mu \mathrm{L}$ of anti-3-NTy antibody for $1 \mathrm{~h}$ at room temperature. The plate was washed with wash buffer and incubated with $100 \mu \mathrm{L}$ of secondary anti-3-NTy antibody for $1 \mathrm{~h}$ at room temperature. Subsequently, $100 \mu \mathrm{L}$ of the substrate solution was added, and the optical absorbance at $450 \mathrm{~nm}$ was determined. The equation of the 3-NTy standard curve was calculated, and the data obtained from the brain samples were interpolated.

\subsection{Statistical Analysis}

The data in the text, tables, and figures are expressed as the mean \pm standard error of the mean (SEM) for 6 animals. All the statistical analyses were performed with the Statistical Package for Social Sciences v25.0 (SPSS Co., Chicago, IL, USA). Unpaired Student's t-tests were used to compare the differences between the means. In all cases, statistical significance was assumed at a value of $p<0.05$.

\section{Results}

In the in vitro model of the hypoxia-reoxygenation of the brain slices of rats, an increase in LDH efflux $(\times 4.6)$, lipid peroxidation (as the brain tissue TBARS concentration) $(\times 7.3)$, and nitrosative stress (as the brain tissue 3-NTy concentration) $(\times 9.0)$ occurred after the reoxygenation period, with respect to the preanoxia values (Table 2 ).

First, we carried out hypoxia-reoxygenation experiments in rat brain sections, incubating with the four polyphenol compounds according to their reported concentrations in EVOO, according to high, medium, or low EVOO polyphenol contents, and compared the results with those for HT alone at the same concentration. Figure 2 shows the results for LDH efflux, TBARS, and 3-nitrotyrosine, expressed as percentages for each variable with respect to the values obtained for the control samples after the reoxygenation period. The cytoprotective and antioxidant effects of the mixture of HT, Ty, DHPG, and OLC were 
significantly greater than those of HT alone, by $30-50 \%$, except for 3-NTy, which was inhibited to the same extent by HT as by the mixture of polyphenols.

The four polyphenols showed cytoprotective and antioxidant effects and inhibited peroxynitrite production in a concentration-dependent manner (Figure 3). Table 3 shows the concentrations of the compounds that produced $50 \%$ inhibition of each variable after the reoxygenation period $\left(\mathrm{IC}_{50}\right)$. Regarding the cytoprotective and antioxidant effects ( $\mathrm{LDH}$ efflux and TBARS, respectively), HT and OLC showed significantly greater effects ( $\mathrm{IC}_{50}$ in the $10^{-5} \mathrm{M}$ range) than Ty and DHPG ( $\mathrm{IC}_{50}$ in the $10^{-4} \mathrm{M}$ range) did. The effects on the production of peroxynitrite (3-NTy) were similar in all cases $\left(\mathrm{IC}_{50}\right.$ in the range $10^{-5} \mathrm{M}$ ).

Table 2. Mean values (mean \pm SEM) of LDH efflux, thiobarbituric acid reactive substances (TBARS), and 3-nitrotyrosine (3-NTy) in rat brain slices subjected to a hypoxia-reoxygenation process $(\mathrm{N}=6)$.

\begin{tabular}{ccc}
\hline- & Pre-Hypoxia & Post-Reperfusion \\
\hline LDH efflux $\left(\mathrm{OD}_{492}-\mathrm{OD}_{600} \mathrm{~nm}\right)$ & $0.7 \pm 0.1$ & $3.2 \pm 0.3^{*}$ \\
TBARS (nmol/mg protein) & $0.8 \pm 0.06$ & $5.9 \pm 0.6^{*}$ \\
3-NTy (nmol/mg tissue) & $0.3 \pm 0.02$ & $2.7 \pm 0.2^{*}$ \\
\hline
\end{tabular}

${ }^{*} p<0.0001$ with respect to pre-hypoxia values.

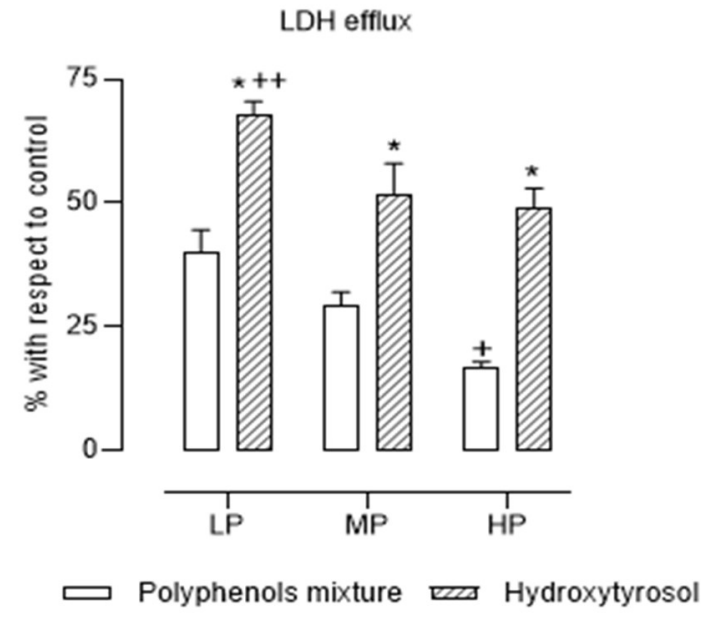

TBARS

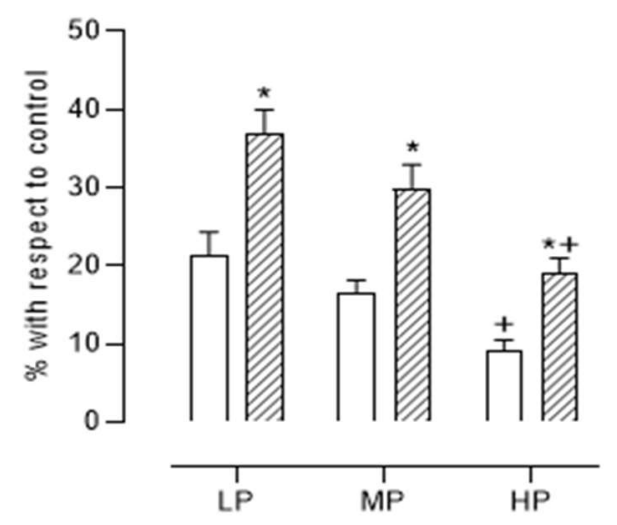

Polyphenols mixture שydroxytyrosol

Figure 2. Cont. 


\section{3-Nitrotyrosine}

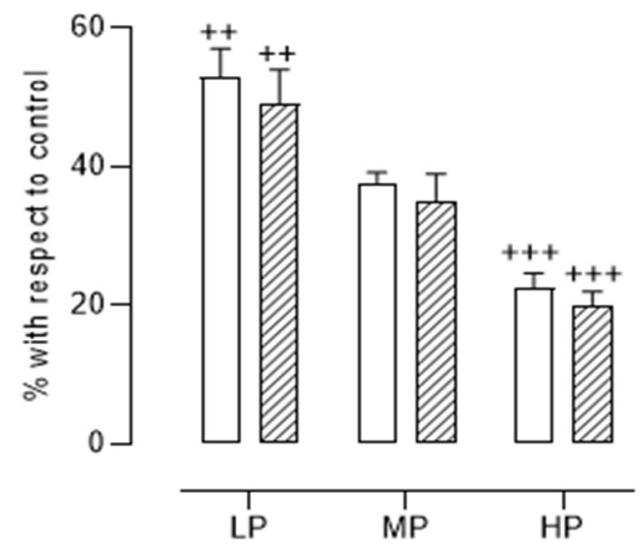

\section{$\varpi$ Polyphenols mixture Hydroxytyrosol}

Figure 2. Mean values (mean \pm SEM) of the percentages with respect to control samples of lactate dehydrogenase efflux (LDH efflux), thiobarbituric acid reactive substances (TBARS), and 3-nitrotyrosine in rat brain slices incubated with hydroxytyrosol, tyrosol, $3^{\prime}, 4^{\prime}$-dihydroxyphenylglycol, and oleocanthal in the same proportions of extra virgin olive oils considered to have low (LP), medium (MP), and high (HP) polyphenol contents (see Table 1$)\left(\mathrm{N}=6\right.$ different experiments). ${ }^{*} p<0.0001$ with respect to polyphenol mixture, $+p<0.05$ with respect to $\mathrm{LP}$ and $\mathrm{MP},++p<0.05$ with respect to $\mathrm{MP}$ and $\mathrm{HP}$, and $+++p<0.05$ with respect to MP.

Table 3. Mean values (mean \pm SEM) of the concentrations that produced a $50 \%$ inhibition with respect to control samples $\left(\mathrm{IC}_{50}, \mu \mathrm{M}\right)$ of lactate dehydrogenase efflux $(\mathrm{LDH})$, thiobarbituric acid reactive substances (TBARS), and 3-nitrotyrosine (3-NTy) in brain tissue after the hypoxia-reoxygenation model ( $\mathrm{N}=6$ different experiments per group).

\begin{tabular}{cccc}
\hline Compound & LDH & TBARS & 3-NTy \\
\hline Hydroxytyrosol $(\mathrm{HT})$ & $81.1 \pm 4.7^{* * * * *}$ & $57.1 \pm 5.7^{* *}$ & $64.4 \pm 5.8$ \\
Tyrosol (Ty) & $425 \pm 25.1$ & $154 \pm 13.3^{* * *}$ & $68.9 \pm 4.4$ \\
DHPG & $314 \pm 22.5$ & $84.7 \pm 6.3$ & $40.5 \pm 3.9^{*}$ \\
Oleocanthal (OLC) & $78.9 \pm 5.8^{* * * * *}$ & $82.0 \pm 6.3$ & $75.3 \pm 6.4$ \\
- & - & - & - \\
HT + Ty $(10 \mu \mathrm{M})$ & $185 \pm 12.6^{* * * *}$ & $51.3 \pm 6.5$ & $71.5 \pm 5.7$ \\
HT + Ty $(50 \mu \mathrm{M})$ & $227 \pm 8.2^{* * * *}$ & $41.7 \pm 3.9$ & $57.6 \pm 4.2$ \\
HT + Ty $(100 \mu \mathrm{M})$ & $125 \pm 11.2^{* * * *}$ & $91.4 \pm 5.0^{* * * *}$ & $84.2 \pm 8.4$ \\
- & - & - & - \\
HT + DHPG $(10 \mu \mathrm{M})$ & $28.3 \pm 3.4^{* * * *}$ & $56.0 \pm 5.2$ & $27.7 \pm 4.8^{* * * *}$ \\
HT + DHPG $(50 \mu \mathrm{M})$ & $85.9 \pm 8^{* * 3}$ & $30.1 \pm 3.8^{* * * *}$ & $29.4 \pm 4.7^{* * * *}$ \\
HT + DHPG $(100 \mu \mathrm{M})$ & $84.3 \pm 8^{* * 2}$ & $49.8 \pm 5.6^{* * * *}$ & $32.9 \pm 4.5^{* * * *}$ \\
- & - & - & - \\
HT + OLC $(10 \mu \mathrm{M})$ & $19.1 \pm 2.6^{* * * *}$ & $34.8 \pm 2.9^{* * * *}$ & $67.2 \pm 7.1$ \\
HT + OLC $(50 \mu \mathrm{M})$ & $21.2 \pm 1.8^{* * * *}$ & $26.0 \pm 2.8^{* * * *}$ & $65.3 \pm 6.6$ \\
HT + OLC $(100 \mu \mathrm{M})$ & $18.3 \pm 1.1^{* * * *}$ & $21.2 \pm 2.7^{* * * *}$ & $68.0 \pm 5.7$ \\
\hline
\end{tabular}

${ }^{*} p<0.05$ with respect to HT, Ty, and OLC. ${ }^{* *} p<0.01$ with respect to Ty, DHPG, and OLC. ${ }^{* * *} p<0.001$ with respect to HY, DHPG, and OLC. ${ }^{* * * *} p<0.005$ with respect to HT. ${ }^{* * * * *} p<0.0001$ with respect to Ty and DHPG.

When increasing concentrations of HT were used in the presence of Ty, the antioxidant and peroxynitrite-production-inhibitory effects were not quantitatively modified, but the cytoprotective effect of HT was reduced (Figure 4 and Table 3). 


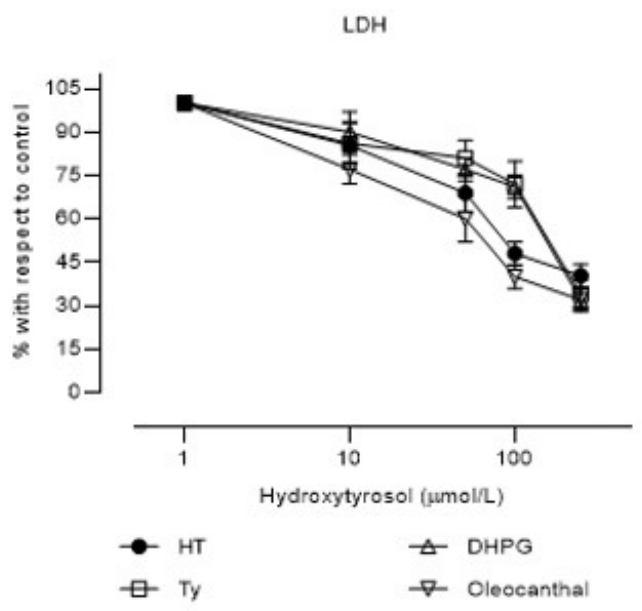

TBARS

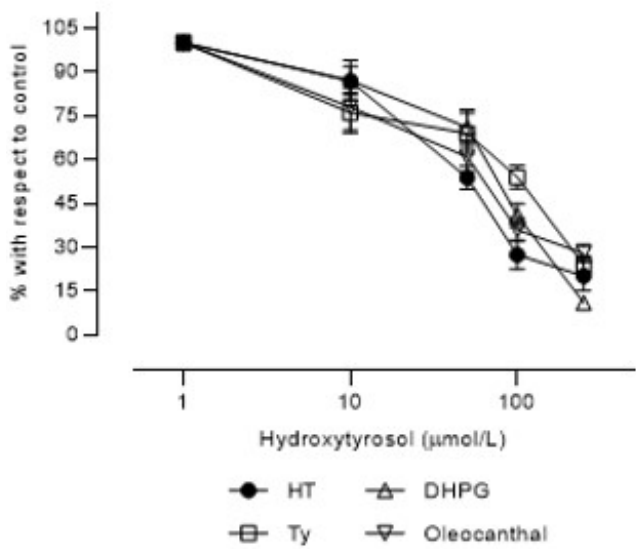

3-Nitrotyrosine

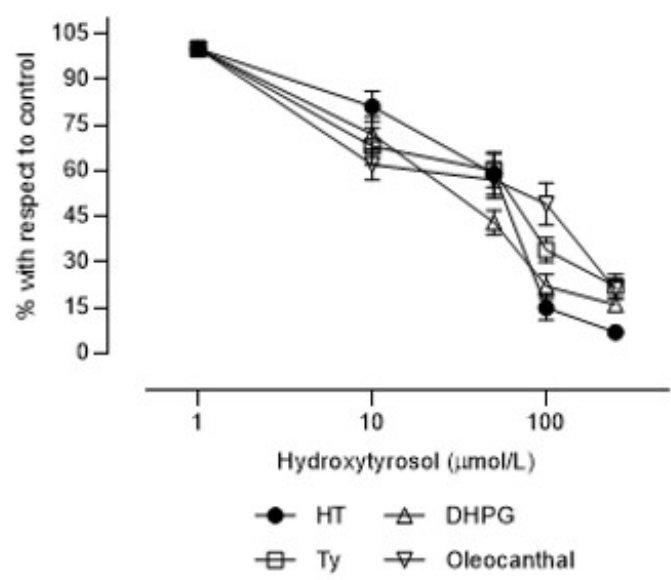

Figure 3. Concentration-effect curves (mean \pm SEM) of the percentages with respect to control samples of lactate dehydrogenase efflux (LDH efflux), thiobarbituric acid reactive substances (TBARS), and 3-nitrotyrosine in rat brain slices incubated with hydroxytyrosol, tyrosol, $3^{\prime}, 4^{\prime}$ dihydroxyphenylglycol, and oleocanthal ( $\mathrm{N}=6$ different experiments). All the compounds were incubated at $1,10,50,100$, and $250 \mu \mathrm{M}$. 


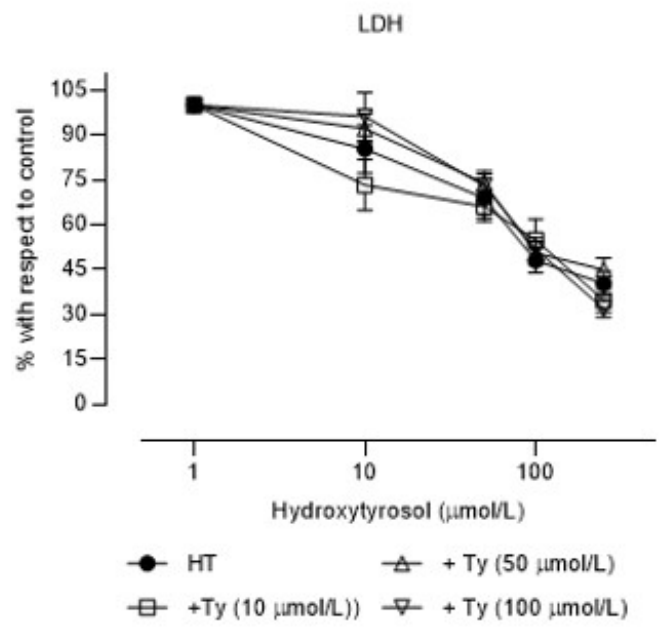

TBARS

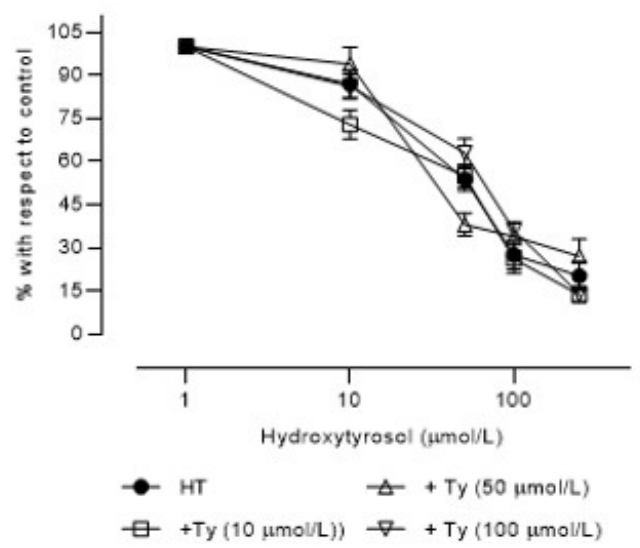

3-Nitrotyrosine

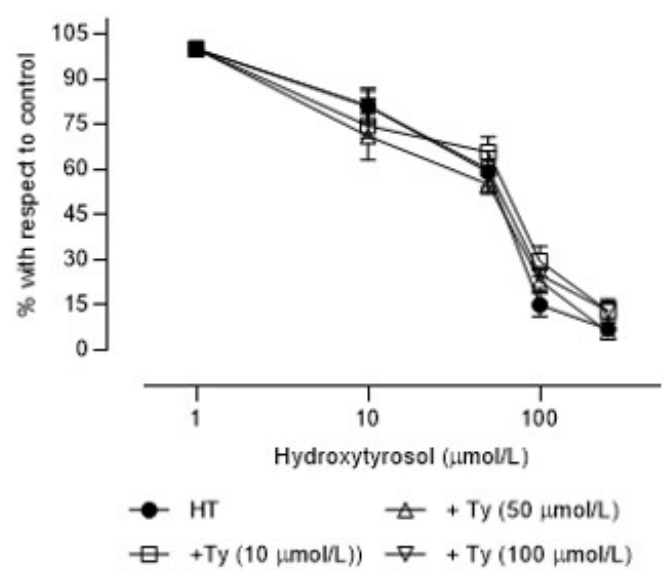

Figure 4. Concentration-effect curves (mean \pm SEM) of the percentages with respect to control samples of lactate dehydrogenase efflux (LDH efflux), thiobarbituric acid reactive substances (TBARS), and 3-nitrotyrosine in rat brain slices incubated with hydroxytyrosol (HT) alone or in the presence of 10,50 , or $100 \mu \mathrm{M}$ tyrosol ( $\mathrm{N}=6$ different experiments).

The concentration-effect curves for HT's cytoprotective effect were shifted to the left (higher power) when $10 \mu \mathrm{M}$ DHPG was present (the $\mathrm{IC}_{50}$ value was $65.1 \%$ lower), as were 
those for the antioxidant effect when 50 and $100 \mu \mathrm{M}$ DHPG was present (the $\mathrm{IC}_{50}$ values were $47.3 \%$ and $12.8 \%$ lower, respectively), and all three concentrations caused leftward shifts for the inhibition of peroxynitrite formation (the $\mathrm{IC}_{50}$ values were $56.9 \%, 54.3 \%$, and $48.9 \%$ lower, respectively) (Figure 5 and Table 3 ).

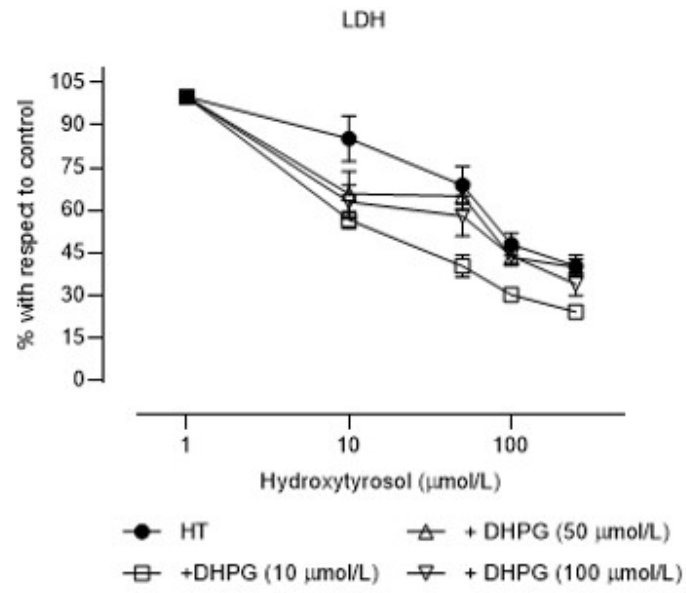

TBARS

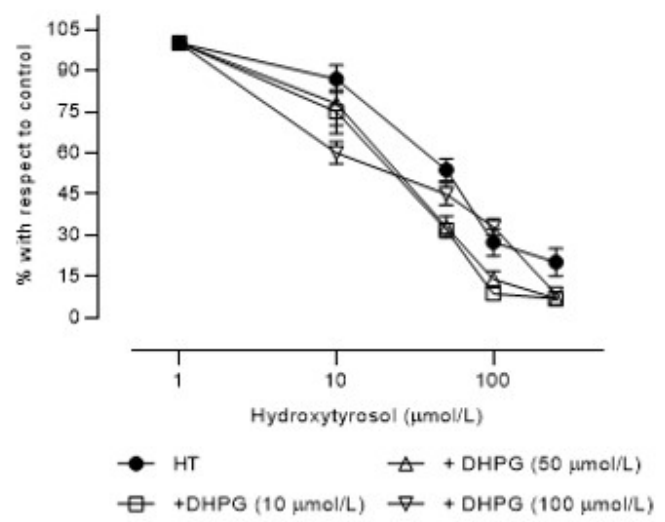

3-Nitrotyrosine

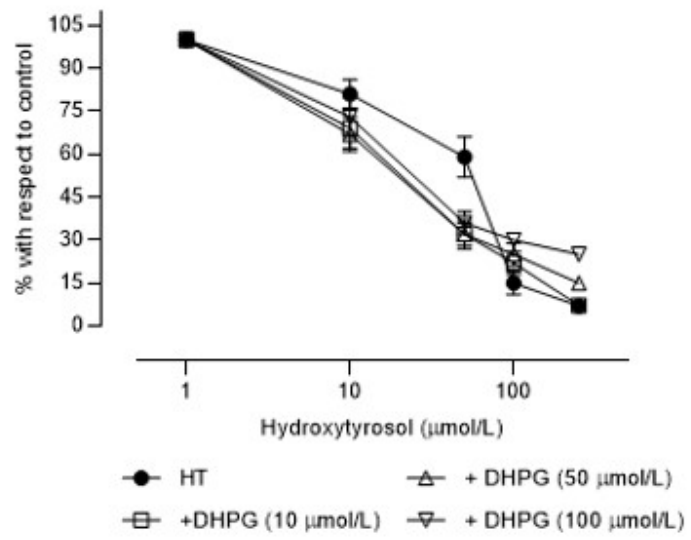

Figure 5. Concentration-effect curves (mean \pm SEM) of the percentages with respect to control samples of lactate dehydrogenase efflux (LDH efflux), thiobarbituric acid reactive substances (TBARS), and 3-nitrotyrosine in rat brain slices incubated with hydroxytyrosol (HT) alone or in the presence of 10,50 , or $100 \mu \mathrm{M} 3^{\prime}, 4^{\prime}$-dihydroxyphenylglycol (DHPG) ( $\mathrm{N}=6$ different experiments). 
The incubation of OLC together with increasing concentrations of HT increased the cytoprotective effect of HT (Figure 6 and Table 3) ( $\mathrm{IC}_{50} 75 \%-80 \%$ lower than those for HT alone). The antioxidant effect of HT was also increased in the presence of OLC (IC 50 $40 \%-60 \%$ lower than those for HT alone). The inhibitory effect of HT on peroxynitrite production was not modified by the presence of OLC.
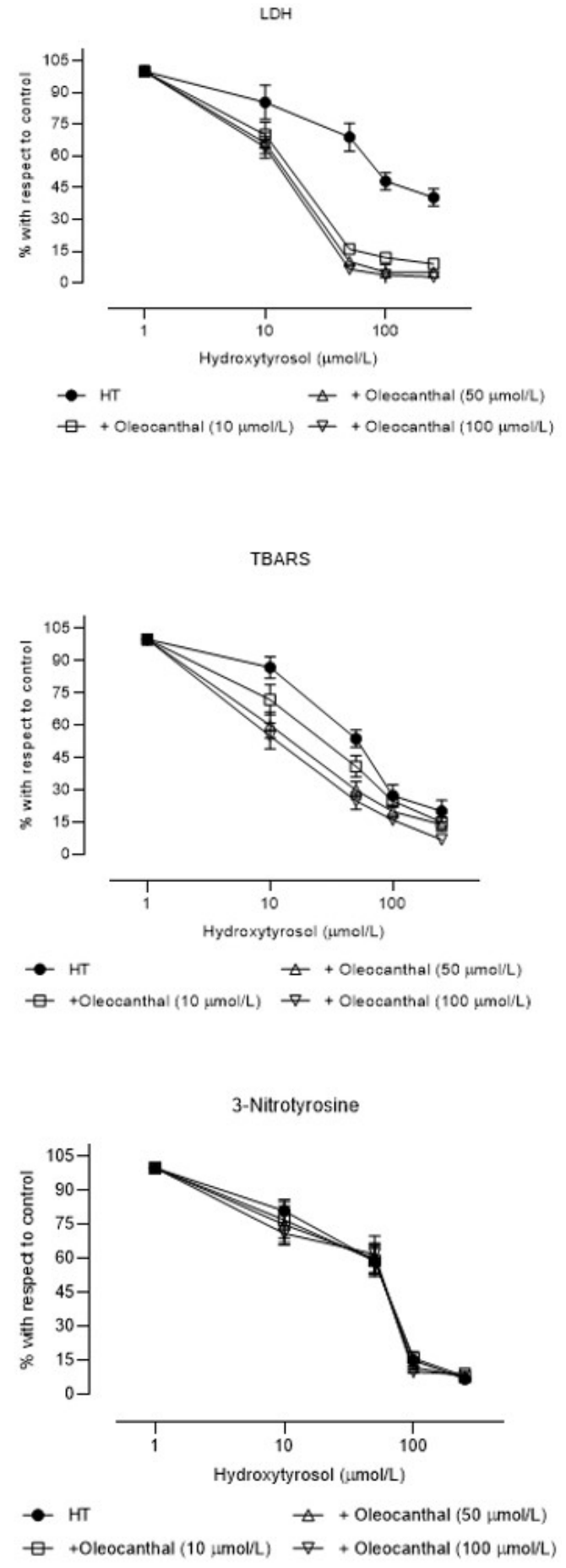

Figure 6. Concentration-effect curves (mean \pm SEM) of the percentages with respect to control samples of lactate dehydrogenase efflux (LDH efflux), thiobarbituric acid reactive substances (TBARS), and 3-nitrotyrosine in rat brain slices incubated with hydroxytyrosol (HT) alone or in presence of 10, 50 , or $100 \mu \mathrm{M}$ oleocanthal ( $\mathrm{N}=6$ different experiments). 


\section{Discussion}

The results obtained in this study show that the main polyphenols of EVOO interact under in vitro conditions in rat brain tissue, mainly in terms of the cytoprotective effect and possibly through an interaction of antioxidant effects and inhibitory effects on the production of peroxynitrite, among other possible mechanisms.

We chose the in vitro hypoxia-reoxygenation model using rat brain slices because it has been shown that, in this model, there is cell death accompanied by an increase in oxidative and nitrosative stress $[16,17]$. This was fulfilled in the present study, as can be seen in Table 2.

It is known that EVOO polyphenols, mainly HT, exert cytoprotective effects in various tissues and experimental models [8,18-20]. These effects are due to various mechanisms of action, but it is accepted that the demonstrated antioxidant effects of these compounds are the main axes of their actions [21]. In the experimental model used in this study, the inhibition of lipid peroxidation by the four compounds was verified (Table 3 and Figure 3), presenting the following order regarding the potency of their antioxidant effects: HT > OLC $\geq$ DHPG $>$ Ty. However, the order in relation to inhibitory effects on peroxynitrite formation was DHP G $>$ HT $\geq$ Ty $\geq$ OLC.

First, we wanted to verify the general hypothesis raised in this study, which was that the cytoprotective effect of HT does not alone explain the cytoprotective effect of EVOO with regard to its content of other polyphenols [13,14]. To confirm this, we incubated the four polyphenols in the same proportions as found within EVOO—for low, medium, and high polyphenol contents - and compared their effects with those of HT alone with the same proportions. We were able to observe that, in all cases, HT showed lower cytoprotective and antioxidant effects than the combination of polyphenols did, except for the inhibition of the production of peroxynitrite, for which HT seemed to carry the highest specific weight (Figure 2). Therefore, it was confirmed that the combination of polyphenols explained the cytoprotective effect of EVOO better than HT alone did.

The four compounds showed cytoprotective effects separately in this experimental model, although HT and OLC showed greater potency than Ty and DHPG did. Using the same model, this effect has been previously described for HT [8,15-17] and Ty $[22,23]$ at the same proportions as in this study. OLC has demonstrated a neuroprotective effect on neuron-like SH-SY5Y cells induced with $\mathrm{H}_{2} \mathrm{O}_{2}$ at proportions similar to those in our study and at similar concentrations [24]. Another study showed a neuroprotective effect of OLC in Alzheimer's models [25]. We found no studies in the consulted literature that demonstrated a neuroprotective effect of DHPG in the chemical form that EVOO contains.

It is indisputable that the antioxidant effects play a fundamental role in the effects of the studied compounds, and there are other mechanisms that possibly participate, such as the anti-inflammatory effects [26]. As can be seen in Table 3, except for those of DHPG, there is a parallel between the $\mathrm{IC}_{50}$ values for the inhibition of lipid peroxidation and the formation of peroxynitrite and LDH efflux.

From a pharmacological point of view, DHPG and OLC increase the cytoprotective effect of HT, while Ty decreases it. Ty has been reported to exert a poor cytoprotective effect in vitro [22], while its ex vivo effect resembles that of HT [23]. One possible explanation is that, in vitro, Ty acts on the same mechanisms as HT does but with much less potency, which would make it a partial agonist, that is, a weak cytoprotective compound if used alone and an inhibitor of the effect of HT when both are present in brain tissue. In these cases, when high concentrations of an $\mathrm{AgP}$ are incubated together with an $\mathrm{Ag}$, the former behaves as an antagonist. In the experiments of this study, it was shown that Ty tended to inhibit the effects of HT as an antioxidant and inhibitor of peroxynitrite production, especially at higher concentrations, which could partially support the hypothesis. Furthermore, it has been described that, under ex vivo conditions, some Ty is transformed into HT in the liver [27], which could also explain the greater effect in this type of experiment [23].

DHPG increases the cytoprotective effect of HT, possibly due to an increase in its effect on the production of peroxynitrite, since its antioxidant effect only increased at the lower 
concentration (Table 3). In EVOO, the concentration of DHPG is usually around $10 \%$ of that of HT [28]. If we extrapolate the results of LDH efflux with $100 \mu \mathrm{M}$ HT and $10 \mu \mathrm{M}$ DHPG, we observe that HT reduced this variable by $63 \%$, DHPG by $9 \%$, and the combination of both by $70 \%$. A synergistic effect between both polyphenols was demonstrated to occur at the level of platelet function and lipid peroxidation in vitro [29].

A stimulating effect on the cytoprotective and antioxidant actions of HT was observed for OLC, although OLC did not modify the effect of HT on the production of peroxynitrite. It was demonstrated that OLC exerted an inhibitory effect on cyclooxygenase (COX) types 1 and 2 [30], which could indirectly decrease the production of lipid peroxides and promote the cytoprotective effect of HT; some nonsteroidal anti-inflammatory drugs have similar effects, especially those that inhibit both types of cyclooxygenases or, to a greater extent, COX-2 [31].

Through pharmacological analysis, the results of this study show which polyphenols in EVOO (DHPG and OLC) increase the cytoprotective effect of HT, which has not been previously demonstrated. However, this study has a fundamental limitation regarding the concentrations used. Our intention was to assess the existence of these interactions, but it is necessary to carry out the same experiments using the concentrations at which these polyphenols are found in brain tissue after the oral administration of an EVOO with a high polyphenol content.

\section{Conclusions}

This study shows that the EVOO polyphenols DHPG and OLC improve the protective effects of hydroxytyrosol in an in vitro model of the hypoxia-reoxygenation of rat brain slices, mainly due to a possibly synergistic effect at the level of its antioxidant action. These results could explain the greater cytoprotective effect of EVOO than of the polyphenols alone. Likewise, they could form the basis for the development of a functionalized oil with higher contents of DHPG and OLC in order to increase the cytoprotective effect of EVOO.

Author Contributions: I.P.d.A.: methodology and investigation; M.D.R.-P.: methodology and investigation; M.M.A.: methodology and investigation; E.M.-A.: validation and formal analysis; L.O.-H.: methodology and investigation; C.V.: methodology and investigation; A.B.-O.: methodology and validation; M.Á.F.-P.: methodology and validation; J.P.D.L.C.C.: conceptualization, formal analysis, investigation, resources, writing - original draft, supervision, and project administration; J.A.G.-C.: conceptualization, formal analysis, investigation, resources, writing-original draft, supervision, and project administration. All authors have read and agreed to the published version of the manuscript.

Funding: This study was supported in part by Consejería de Salud-Junta de Andalucía (Spain), Proyectos de Investigación en Salud (PI-0129-2017), and by Ministerio de Economía y Competitividad (Spain), Centro para el Desarrollo Tecnológico Industrial, Programa FEDER Interconecta PluriRegional (PS17173. NUTRADAF, ITC-20161265).

Institutional Review Board Statement: All the rats were used in accordance with the current Spanish legislation for animal care, use and housing (EDL 2013/80847, BOE-A-2013-6271). The recommendations of the Guide for the Care and Use of Laboratory Animals (NIH publication No. 86-23, revised 1985) were followed, as was the Spanish Law on the Protection of Animals, where applicable. The study protocol was approved by the University of Malaga Ethics Committee for the Use of Animals (Ref. CEUMA31-2018-A) and the Consejería de Agricultura, Ganadería, Pesca y Desarrollo Sostenible, Junta de Andalucía (Department of Agriculture, Livestock, Fisheries and Sustainable Development of the Regional Government of Andalusia) (Ref. 9/07/2019/124).

Acknowledgments: We thank Ana Guerrero and Nadia Valverde for their excellent technical assistance.

Conflicts of Interest: The authors do not declare any conflict of interest. 


\section{References}

1. Martínez-González, M.A.; Salas-Salvadó, J.; Estruch, R.; Corella, D.; Fitó, M.; Ros, E.; PREDIMED Investigators. Benefits of the Mediterranean diet: Insights from the PREDIMED Study. Prog. Cardiovasc. Dis. 2015, 58, 50-60. [CrossRef]

2. Lasa, A.; Miranda, J.; Bulló, M.; Casas, R.; Salas-Salvadó, J.; Larretxi, I.; Estruch, R.; Ruiz-Gutiérrez, V.; Portillo, M.P. Comparative effect of two Mediterranean diets versus a low-fat diet on glycaemic control in individuals with type 2 diabetes. Eur. J. Clin. Nutr. 2014, 68, 767-772. [CrossRef]

3. De La Cruz, J.P.; Del Río, S.; Arrebola, M.M.; López-Villodres, J.A.; Jebrouni, N.; González-Correa, J.A. Effect of virgin olive oil plus acetylsalicylic acid on brain slices damage after hypoxia-reoxygenation in rats with type 1-like diabetes mellitus. Neurosci. Lett. 2010, 471, 89-93. [CrossRef]

4. González-Correa, J.A.; Muñoz-Marín, J.; Arrebola, M.M.; Guerrero, A.; Narbona, F.; López-Villodres, J.A.; De La Cruz, J.P. Dietary virgin olive oil reduces oxidative stress and cellular damage in rat brain slices subjected to hypoxia-reoxygenation. Lipids 2007, 42, 921-929. [CrossRef]

5. Garcia-Martinez, O.; Ruiz, C.; Gutierrez-lbanez, A.; Illescas-Montes, R.; Melguizo-Rodriguez, L. Benefits of olive oil phenolic compounds in disease prevention. Endocr. Metab. Immune Disord. Drug Targets 2018, 18, 333-340. [CrossRef]

6. Rodríguez-Morató, J.; Xicota, L.; Fitó, M.; Farré, M.; Dierssen, M.; de la Torre, R. Potential role of olive oil phenolic compounds in the prevention of neurodegenerative diseases. Molecules 2015, 20, 4655-4680. [CrossRef]

7. Robles-Almazan, M.; Pulido-Moran, M.; Moreno-Fernandez, J.; Ramirez-Tortosa, C.; Rodriguez-Garcia, C.; Quiles, J.L.; Ramirez-Tortosa, M. Hydroxytyrosol: Bioavailability, toxicity, and clinical applications. Food Res. Int. 2018, 105, 654-667. [CrossRef] [PubMed]

8. Cabrerizo, S.; De La Cruz, J.P.; López-Villodres, J.A.; Muñoz-Marín, J.; Guerrero, A.; Reyes, J.J.; Labajos, M.T.; González-Correa, J.A. Role of the inhibition of oxidative stress and inflammatory mediators in the neuroprotective effects of hydroxytyrosol in rat brain slices subjected to hypoxia reoxygenation. J. Nutr. Biochem. 2013, 24, 2152-2157. [CrossRef] [PubMed]

9. Jimenez-Lopez, C.; Carpena, M.; Lourenço-Lopes, C.; Gallardo-Gomez, M.; Lorenzo, J.M.; Barba, F.J.; Prieto, M.A.; Simal-Gandara, J. Bioactive compounds and quality of extra virgin olive oil. Foods 2020, 9, 1014. [CrossRef]

10. Borges, T.H.; Serna, A.; López, L.C.; Lara, L.; Nieto, R.; Seiquer, I. Composition and antioxidant properties of Spanish extra virgin olive oil regarding cultivar, harvest year and crop stage. Antioxidants 2019, 8, 217. [CrossRef] [PubMed]

11. Rubio-Senent, F.; Rodríguez-Gutiérrez, G.; Lama-Muñoz, A.; Fernández-Bolaños, J. New phenolic compounds hydrothermally extracted from the olive oil by-product alperujo and their antioxidative activities. J. Agric. Food Chem. 2012, 60, 1175-1186. [CrossRef]

12. Fernández-Bolaños, J.; Rodríguez, G.; Rodríguez, R.; Heredia, A.; Guillén, R.; Jiménez, A. Production in large quantities of highly purified hidroxitirosol from liquid-solid waste of two-phase olive oil processing or "Alperujo". J. Agric. Food Chem. 2002, 50, 6804-6811. [CrossRef]

13. Pedan, V.; Popp, M.; Rohn, S.; Nyfeler, M.; Bongartz, A. Characterization of phenolic compounds and their contribution to sensory properties of olive oil. Molecules 2019, 24, 2041. [CrossRef]

14. Medina, E.; de Castro, A.; Romero, C.; Brenes, M. Comparison of the concentrations of phenolics compounds in olive oils and other plant oils: Correlation with antimicrobial activity. J. Agric. Food Chem. 2006, 54, 4954-4961. [CrossRef]

15. Guerrero, A.; De la Cruz, J.P.; Muñoz-Marín, J.; López-Villodres, J.A.; Madrona, A.; Espartero, J.L.; González-Correa, J.A. Neuroprotective effect of alkyl hydroxytyrosyl ethers in rat brain slices subjected to a hypoxia-reoxygenation model. Food Chem. 2012, 134, 2176-2183. [CrossRef]

16. González-Correa, J.A.; Arrebola, M.M.; Cansino, A.L.; Muñoz-Marín, J.; Ruiz-Villafranca, D.; Guerrero, A.; Sánchez de la Cuesta, F.; De La Cruz, J.P. Effects of hypoxia reoxygenation in brain slices from rats with type 1-like diabetes mellitus. Diabetes Metab. Res. Rev. 2006, 22, 390-400. [CrossRef]

17. Reyes, J.J.; Villanueva, B.; López-Villodres, J.A.; De La Cruz, J.P.; Romero, L.; Rodríguez-Pérez, M.D.; Rodriguez-Gutierrez, G.; Fernández-Bolaños, J.; González-Correa, J.A. Neuroprotective effect of hydroxytyrosol in experimental diabetes mellitus. J. Agric. Food Chem. 2017, 65, 4378-4383. [CrossRef]

18. Echeverría, F.; Ortiz, M.; Valenzuela, R.; Videla, L.A. Hydroxytyrosol and cytoprotection: A projection for clinical interventions. Int. J. Mol. Sci. 2017, 18, 930. [CrossRef]

19. Pan, S.; Liu, L.; Pan, H.; Ma, Y.; Wang, D.; Kang, K.; Wang, J.; Sun, B.; Sun, X.; Jiang, H. Protective effects of hydroxytyrosol on liver ischemia/reperfusion injury in mice. Mol. Nutr. Food Res. 2013, 57, 1218-1227. [CrossRef]

20. Tejada, S.; Pinya, S.; Del Mar Bibiloni, M.; Tur, J.A.; Pons, A.; Sureda, A. Cardioprotective effects of the polyphenol hydroxytyrosol from olive oil. Curr. Drug Targets 2017, 18, 1477-1486. [CrossRef]

21. Beccari, T.; Michelini, S. Hydroxytyrosol: A natural compound with promising pharmacological activities. J. Biotechnol. 2020, 309, 29-33.

22. De La Cruz, J.P.; Ruiz-Moreno, M.I.; Guerrero, A.; López-Villodres, J.A.; Reyes, J.J.; Espartero, J.L.; Labajos, M.T.; González-Correa, J.A. Role of the catechol group in the antioxidant and neuroprotective effects of virgin olive oil components in rat brain. J. Nutr. Biochem. 2015, 26, 549-555. [CrossRef]

23. De La Cruz, J.P.; Ruiz-Moreno, M.I.; Guerrero, A.; Reyes, J.J.; Benitez-Guerrero, A.; Espartero, J.L.; González-Correa, J.A. Differences in the neuroprotective effect of orally administered virgin olive oil (Olea europaea) polyphenols tyrosol and hydroxytyrosol in rats. J. Agric. Food Chem. 2015, 63, 5957-5963. [CrossRef] [PubMed] 
24. Giusti, L.; Angeloni, C.; Barbalace, M.C.; Lacerenza, S.; Ciregia, F.; Ronci, M.; Urbani, A.; Manera, C.; Digiacomo, M.; Macchia, M.; et al. A proteomic approach to uncover neuroprotective mechanisms of oleocanthal against oxidative stress. Int. J. Mol. Sci. 2018, 19, 2329. [CrossRef] [PubMed]

25. Grewal, R.; Reutzel, M.; Dilberger, B.; Hein, H.; Zotzel, J.; Marx, S.; Tretzel, J.; Sarafeddinov, A.; Fuchs, C.; Eckert, G.P. Purified oleocanthal and ligstroside protect against mitochondrial dysfunction in models of early Alzheimer's disease and brain ageing. Exp. Neurol. 2020, 328, 113248. [CrossRef]

26. Bucciantini, M.; Leri, M.; Nardiello, P.; Casamenti, F.; Stefani, M. Olive polyphenols: Antioxidant and anti-inflammatory properties. Antioxidants 2021, 10, 1044. [CrossRef] [PubMed]

27. Rodríguez-Morató, J.; Robledo, P.; Tanner, J.A.; Boronat, A.; Pérez-Mañá, C.; Oliver Chen, C.Y.; Tyndale, R.F.; de la Torre, R. CYP2D6 and CYP2A6 biotransform dietary tyrosol into hydroxytyrosol. Food Chem. 2017, 217, 716-725. [CrossRef]

28. Fernández-Prior, M.Á.; Fatuarte, J.C.P.; Oria, A.B.; Viera-Alcaide, I.; Fernández-Bolaños, J.; Rodríguez-Gutiérrez, G. New liquid source of antioxidant phenolic compounds in the olive oil industry: Alperujo water. Foods 2020, 9, 962. [CrossRef] [PubMed]

29. Rubio-Senent, F.; de Roos, B.; Duthie, G.; Fernández-Bolaños, J.; Rodríguez-Gutiérrez, G. Inhibitory and synergistic effects of natural olive phenols on human platelet aggregation and lipid peroxidation of microsomes from vitamin E-deficient rats. Eur. J. Nutr. 2015, 54, 1287-1295. [CrossRef]

30. Beauchamp, G.K.; Keast, R.S.; Morel, D.; Lin, J.; Pika, J.; Han, Q.; Lee, C.H.; Smith, A.B.; Breslin, P.A. Phytochemistry: Ibuprofenlike activity in extra-virgin olive oil. Nature 2005, 437, 45-46. [CrossRef]

31. López-Villodres, J.A.; De La Cruz, J.P.; Muñoz-Marin, J.; Guerrero, A.; Reyes, J.J.; González-Correa, J.A. Cytoprotective effect of nonsteroidal antiinflammatory drugs in rat brain slices subjected to reoxygenation after oxygen-glucose deprivation. Eur. $J$. Pharm. Sci. 2012, 45, 624-631. [CrossRef] [PubMed] 